\title{
Editorial
}

\section{Contribution from Cancer Epidemiological Studies in China}

\author{
Wei-cheng You ${ }^{*}$ \\ The Key Laboratory of Carcinogenesis and Translational Research (Ministry of Education), Department of Cancer Epidemiology, \\ Peking University School of Oncology, Beijing Cancer Hospital \& Institute, Beijing 100142, China
}

DOI: $10.1007 / \mathrm{s} 11670-011-0001-y$

(C) Chinese Anti-Cancer Association and Springer-Verlag Berlin Heidelberg 2011

In this issue, we published a few article of epidemiological studies in China, including data on cancer incidence and mortality nationwide in 2006, 10 -year time trends of cancer in Beijing, prevalence of HPV and cervical intraepithelial neoplasia among young women, and plasma vitamin D levels and its receptor polymorphisms in association with survival of non-small cell lung cancer, etc. We hope that our readers will be interested in the results of their studies.

With over 1.3 billion people, China has the largest population in the world consisting of 56 different ethnic groups. There is tremendous diversity of environmental conditions, and cancer death rates of various sites vary greatly from one place to another. For example, the world aged-adjusted incidence rate of nasopharynx cancer, an extremely rare cancer worldwide, was 33.96 per 100,000 men in Sihui County, Guangdong Province of southern China compared with 1.08 per 100,000 men in Beijing of northern China. It is the size and diversity which makes China an ideal country in which to conduct large-scale epidemiological studies.

Indeed, in late 1970's, Chinese investigators conducted a population-based mortality survey on all causes of death in 2,392 counties of 29 provinces, municipalities, and autonomous regions of China to display the geography of cancer and explore the causes of cancer ${ }^{1]}$. A map of cancer mortality from 1973-1975 by counties published in 1980 revealed the distribution of cancer rates surprisingly varied in China. We were first time to know the gastric cancer was leading cause of death among all cancers and the rate of nasopharynx cancer exceeded by 30 -fold in high-risk area in the south than low-risk area in the north.

Based on this survey, Chinese scientists initiated the case-control studies of gastric, esophagus, liver, colorectal, cervical, oral cavity, nasopharynx and lung cancers in the areas with exceptionally high prevalence rates of these cancers in early 1980's. Numerous data from those epidemiological

\footnotetext{
*Prof. Weicheng You is Chairman of Chinese Society of Cancer Epidemiology and Fellow of American College of Epidemiology.

E-mail: weichengyou@yahoo.com
}

protective factors for cancers, leading to further investigations had released clues of risk and precancerous lesions and nature history, intervention trial and molecular studies for cancers. In this editorial, only comprehensive studies of gastric and cervical cancers in China in the past 30 years are presented.

Gastric cancer is the second most common cancer in China. Some of the highest gastric cancer death rates are found in Linqu County, a rural area in Shandong Province. Little was known why gastric cancer accounted $40 \%$ of all cancer death in this area. A population-based case-control study was initiated in 1984[2]. Sour pancakes (a local favorite), salted foods, cigarette smoking and a family history of gastric cancer were risk factors, whereas fresh vegetables and an intake of vitamin $\mathrm{C}$ and calcium were most inversely associated with the risk of gastric cancer. This protective effect was more pronounced for vegetables, with those in the highest quartile of intake at less than $50 \%$ of the risk of those in the lowest. A negative association with the risk of gastric cancer, in a dose-response pattern, was found for persons who consumed alliums; that is, garlic, onions and Chinese chives ${ }^{[3]}$.

An investigation of gastric histopathology of 3400 adults in Linqu indicated only less than $2 \%$ of the population had normal gastric mucosa or superficial gastritis. Gastric intestinal metaplasia and dysplasia accounted $33 \%$ and $20 \%$, respectively ${ }^{[4]}$. Follow-up study showed the risk increased for gastric cancer was 29.3 for intestinal metaplasia, 25.8 for mild dysplasia and 104.2 for moderate/severe dysplasia[ ${ }^{[5]}$. H.pylori was associated with an increased risk of dysplasia or gastric cancer and the risk of progression was decreased among subjects with baseline ascorbic acid concentrations in the highest tertile compared with those in the lowest tertile.

An increased risk of gastric cancer in subjects with COX-2-1195AA genotype and H.pylori infection or smoking was found. A significant association between IL-8-251， IL-10-1082, XRCC1-399 or OGG1-326 polymorphism and gastric cancer risk was observed[6]. The p16 and RUNX3 methylation were also associated with H.pylori infection, risk of precancerous gastric lesions and gastric cancer. These findings indicated genetic factors may play an important role in gastric 
carcinogenesis.

Based on the evidences gathered in Linqu, two randomized, placebo-controlled intervention trials had been conducted, which revealed a significant reduction in the prevalence of precancerous lesions as well as favorable effects on gastric cancer after eradication of H.pylori or inhibition of COX-2 expression[7]. Currently, a large trial of among 200,000 adults in Linqu has been undergoing in Linqu to finally approve the hypotheses that gastric cancer can be prevented by H.pylori eradication.

Another important public health issue is cervical cancer, particularly in some rural regions, such as Yangcheng, Shanxi Province. The aged-adjusted incidence rate was 81 per 100,000 women in this area compared with a rate of national average of 9.6 per 100,000 women ${ }^{[8]}$. High-risk HPV infection is believed to be a causal risk for cervical cancer. The population-based surveys conducted in high-risk regions of China revealed the overall prevalence of high-risk HPV was 20.8\% among women aged 35-45 years, 12.2 to $11.7 \%$ among women aged $15-59$ years ${ }^{[9]}$. HPV 16 and HPV 18 were the most common types in cervical cancer, together accounting for $84.5 \%$ of SCC. In addition, the prevalence of HPV-16 was dominated among women with cervical lesions 2 or worse lesions $(68.7 \%)^{[10]}$.

Similar to other parts of the world, studies in China have shown that the women who reported to have multiple sexual partners are at high-risk of HPV infection and cervical cancer. The 6-year follow-up of a screening cohort showed that the relative risk for CIN2+ was 52 among baseline HPV-positive women compared to baseline HPV-negative women ${ }^{[11]}$. As an early detection and screening program of Ministry of Health, P.R. China, from 2 demonstration projects to 42 county sites have been established throughout of China aiming cervical cancer early detection and treatment of cervical precancerous lesions ${ }^{[12]}$. In 17 population-based studies conducted in China, the high-risk HPV DNA test provided strong support to the accuracy and feasibility in cervical cancer screening programs in China, as well as in other similar developing countries ${ }^{[13]}$.

In this issue, a population-based screening study among young Chinese women aged 18-25 years showed that $3.4 \%$ of them were diagnosed with CIN grade 2 or worse lesions and $17.1 \%$ were positive for HPV infection. The good news is that $98.4 \%$ of young women are willing to receive HPV vaccination and would recommend their daughters to be vaccinated after an education. This brings the hope that the cervical cancer may be eliminated in human in the future.
Once again, as Dr. Fraumeni at the National Cancer Institute USA, a long time supporter of collaboration between Chinese and American scientists, said "there are striking variation in incidence of various cancers and several extremely high-risk population, which provide a tremendous opportunity for case-control and intervention studies" during an interview with him and myself before an intervention trial was initiated in Linqu and late an article regarding this trial appeared in a special report in "Science" in 1995.

Finally, I would like to take this opportunity, on behalf of a group of epidemiologists in China, to express deep thanks to all our collaborators in America and many other countries, such as Drs. J Fraumine, W. Blot, P. Taylor, M. Gail, C.S. Yang, P. Correa, P Sipponen, M Samloff, M Blaser and many more.

\section{REFERENCES}

1. Cancer Preventive and Control Office, Ministry of Health. The study on cancer death survey in Chinese population. Beijing: China: People's Health Press, 1979.

2. You WC, Blot WJ, Chang YS, et al. Diet and the high risk of stomach cancer in Shandong, China. Cancer Res 1988; 48:3518-23.

3. You WC, Blot WJ, Chang YS, et al. Allium vegetables and reduced risk of stomach cancer. J Natl Cancer Inst 1989; 81:162-4.

4. You WC, Blot WJ, Li JY, et al. Precancerous gastric lesions in a population at high risk of stomach cancer. Cancer Res 1993; 53:1317-21.

5. You WC, Li JY, Blot WJ, et al. Evolution of precancerous lesions in a rural Chinese population at high risk of gastric cancer. Int J Cancer 1999; 83:615-9.

6. Liu F, Pan K, Zhang X, et al. Genetic variants in cyclooxygenase-2: Expression and risk of gastric cancer and its precursors in a Chinese population. Gastroenterol 2006; 130:1975-84.

7. You WC, Brown LM, Zhang L, et al. Randomized double-blind factorial trial of three treatments to reduce the prevalence of precancerous gastric lesions. J Natl Cancer Inst 2006; 98:974-83.

8. Zhang SW, Chen WQ, Kong LZ, et al. An analysis of cancer incidence and mortality from 30 cancer registries in China, 1998-2002. Bulletin Chin Cancer (in Chinese) 2006; 15:439-48.

9. Li LK, Dai M, Clifford GM, et al. Human papillomavirus infection in Shenyang City, People's Republic of China: A population-based study. Br J Cancer 2006; 95:1593-7.

10. Chen W, Zhang X, Molijn A, et al. Human papillomavirus type-distribution in cervical cancer in China: the importance of HPV 16 and 18. Cancer Causes Control 2009; 20:1705-13.

11. Shi JF, Belinson JL, Zhao FH, et al. Human papillomavirus testing for cervical cancer screening: results from a 6-Year prospective study in rural China. Am J Epidemiol 2009; 170:708-16.

12. Shi JF, Qiao YL, Smith JS, et al. Epidemiology and prevention of human papillomavirus and cervical cancer in China and Mongolia. Vaccine 2008; 26:M53-9.

13. Qiao YL, Sellors JW, Eder PS, et al. A new HPV-DNA test for cervical-cancer screening in developing regions: a cross-sectional study of clinical accuracy in rural China. Lancet Oncol 2008; 9:929-36. 